\title{
TMS Over Dorsolateral Prefrontal Cortex Affects the Timing of Motor Imagery but not Overt Action:
}

\section{Further Support for the Motor-Cognitive Model}

\author{
Marie Martel $^{1}$, Scott Glover ${ }^{1}$ \\ ${ }^{1}$ Department of Psychology, Royal Holloway University of London
}

Running title: DLPFC AND MOTOR IMAGERY

Corresponding author:

Dr. Marie Martel

Dept. of Psychology

Royal Holloway University of London

Egham, Surrey, TW20 0EX

Marie.martel@rhul.ac.uk 


\begin{abstract}
The Motor-Cognitive model suggests a functional dissociation between motor imagery and overt action, in contrast to the traditional notion of shared processes central to the Functional Equivalence view. According to the Motor-Cognitive model, motor imagery differs from overt action primarily through the use of executive resources to monitor and elaborate a motor image during execution. This can result in a lack of correspondence between motor imagery and its overt action counterpart. The present study examined the importance of executive resources in motor imagery using TMS to impair the function of the dorsolateral prefrontal cortex, while measuring the time to complete imagined versus overt actions. In two experiments, TMS over the dorsolateral prefrontal cortex slowed motor imagery but did not affect overt actions. TMS over the same region also interfered with performance of a mental calculation task, though it did not reliably affect less demanding cognitive tasks also thought to rely on executive functions. Taken together, these results were consistent with the MotorCognitive model but not with the idea of functional equivalence. The implications of these results for the theoretical understanding of motor imagery, and potential applications of the Motor-Cognitive model to the use of motor imagery in training and rehabilitation, are discussed.
\end{abstract}

\title{
Keywords
}

Motor imagery; Motor-cognitive model; Functional equivalence; Executive functions; Reaching and grasping 


\section{Introduction}

Humans have a remarkable ability to mentally simulate their own actions. This ability, referred to as motor imagery, is used widely as a technique in motor training and skill learning (see for review $[1,2]$ ), as well as neurological rehabilitation (see for review [3-7]). Given its theoretical and applied value, it is important to develop and evaluate competing theories of motor imagery [8-10]. Two such models that will be tested in the present study are the Motor-Cognitive model $[11,12]$ and the Functional Equivalence view [13-15]

In the Motor-Cognitive model, both motor imagery and overt actions involve distinct planning and execution stages. Prior to initiation, the planning of both behaviours relies heavily on internal motor representations. Here, an appropriate motor programme is selected based on information originating from the environment and the body and forms the basis of a forward model [16-20]. During execution, however, the processes underlying motor imagery and overt action diverge. In an overt action, predictions of the consequences of the movement are compared with sensory feedback obtained during its execution, and unconscious online sensory feedback is used to monitor and, if need be, correct the movement online $[19,20]$. In contrast, in the unfolding of motor imagery, no such sensory feedback is available. Instead, the execution of motor imagery is held to involve the conscious monitoring and elaboration of the unfolding motor image, a process that depends heavily on executive resources $[11,12]$.

A key prediction of the Motor-Cognitive model is that motor imagery should be highly sensitive to interference with executive resources, whereas overt actions should be relatively immune. This prediction has been upheld in previous work by Glover and colleagues [11,12]. There, participants were required to perform interference tasks heavily dependent on executive resources, including counting backwards by threes and generating words from a single letter, while simultaneously executing or imagining an action. In line with the Motor-Cognitive model, both calculation and word generation lengthened imagined movement times much more than overt movement times $[11,12]$. 
Glover and colleagues argued that the interference tasks used executive resources normally engaged by the motor imagery system, resulting in the latter being delayed in generating imagined movements.

Executive functions are heavily associated with the dorsolateral prefrontal cortex (DLPFC), which has been linked with such processes as directing attention, working memory, and inhibition [21-28]. If the Motor-Cognitive model is correct, DLPFC involvement should be important in motor imagery, and its involvement should be greater the greater the reliance on executive resources, such as in the motor imagery of actions for which detailed planning representations do not exist. This notion is consistent with previous studies. For one, numerous studies have observed a greater activation in the DLPFC during motor imagery than overt action [18,29-38], with this activation being greater for novel movements $[34,39]$. For another, motor imagery training has been shown to improve working memory through an increase in prefrontal cortical activity [40], and coupling from Supplementary Motor Area to DLPFC is critical during motor imagery $[41,42]$, with higher connectivity at resting-state predicting greater performance $[43,44]$.

In contrast to the Motor Cognitive model, the Functional Equivalence view of motor imagery suggests a strong neural and behavioural correspondence between overt and imagined actions (e.g. $[13,45,46])$. Much early evidence supported this view. For example, both imagined and overt actions have been shown to follow Fitts law $[35,45,47-50]$. Both also responded similarly to biomechanical constraints imposed by a movement, such as adapting the body posture [51], correctly predicting inertial resistance [52,53], or adapting to the orientation of the object [54-56]. However, other studies observed numerous discrepancies between imagined and overt actions. For example, imagined movements can take either less time [57-60], or more time [11,12,48,57,60-66], than their overt counterparts. Further, macroscopic elements of imagined and overt movements may sometimes differ [67]. The predicted neural equivalence between motor imagery and overt actions in the Functional Equivalence model is also open to question (see for review, [31]). Although much overlap exists, numerous differences in the brain activity and connectivity of overt actions versus motor imagery are evident, including but not limited to the discrepancies in activity in the DLPFC noted above $[41,68-$ 73]. 
In the present study we tested an important prediction of the Motor-Cognitive model that directly conflicts with a core principle of the Functional Equivalence view. Here, we used Transcranial Magnetic Stimulation (TMS) to disrupt the DLPFC in two experiments involving imagined and overt reaching and grasping tasks similar to those used in Glover and colleagues [11,12]. In the first experiment, participants had to execute and imagine executing a movement before and after receiving TMS or sham TMS. In the second experiment, participants had to either execute or imagine executing a movement after receiving TMS or Sham, with or without performing a simultaneous interference task designed to draw on executive resources. In each experiment, we independently tested the effects of the TMS on the executive functions of working memory, inhibition, and task switching measured through a variety of computer-based cognitive tests. We also tested its effects on performance in the calculation task of Experiment 2.

Within the Motor-Cognitive framework, disrupting DLPFC activity should have deleterious effects on executive functions that delay the execution of motor imagery while leaving overt actions relatively unaffected. Conversely, within the Functional Equivalence framework, both overt actions and motor imagery ought to be similarly affected by all variables. The critical and novel test of the two theories thus revolved around the interaction between the variables of TMS and Action (overt action vs. motor imagery). To anticipate, in both experiments we observed that the application of TMS over the DLPFC affected motor imagery but not overt action, supporting the Motor-Cognitive model but not the Functional Equivalence view.

\section{Experiment 1}

\section{Methods}

\section{Participants}

We enrolled 32 participants (29 females, age range 18-32 years old) from the Royal Holloway Department of Psychology undergraduate research participation pool. All participants were righthanded by self-report, had normal or corrected-to-normal vision, had no motor or neurological 
impairments, and were naïve as to the exact purpose of the experiment. All passed the screening test for contraindications to TMS based on the guidelines from Rossi and collaborators [74]. Participants were randomly assigned to either the TMS or Sham group. Each participant gave written informed consent for the study, which was approved by the College Ethics Committee at Royal Holloway, and received course credits in return for participation. Three participants had to be replaced due to technical issues or because they failed to follow the instructions. Two further participants were excluded from the sample during data analysis, leaving a total of 30 participants.

\section{Procedure}

The procedure was organised into three main phases (see Figure 1): First, a PRE-stimulation phase that involved completion of the cognitive and action tasks. Second, application of TMS/Sham over the DLPFC. Third, a repeat of the same cognitive and action tasks POST TMS/Sham. The cognitive tasks were always conducted in the following order: Spatial span, Go/NoGo and Switching. All participants took part in all phases of testing.

Each participant performed both the overt action and motor imagery tasks, both PRE and POST TMS/sham, with order of task counterbalanced across participants following an ABBA design. For both action tasks, participants performed both "tossing" and "placing" actions (see below); the order of these was also counterbalanced using an AABB design.

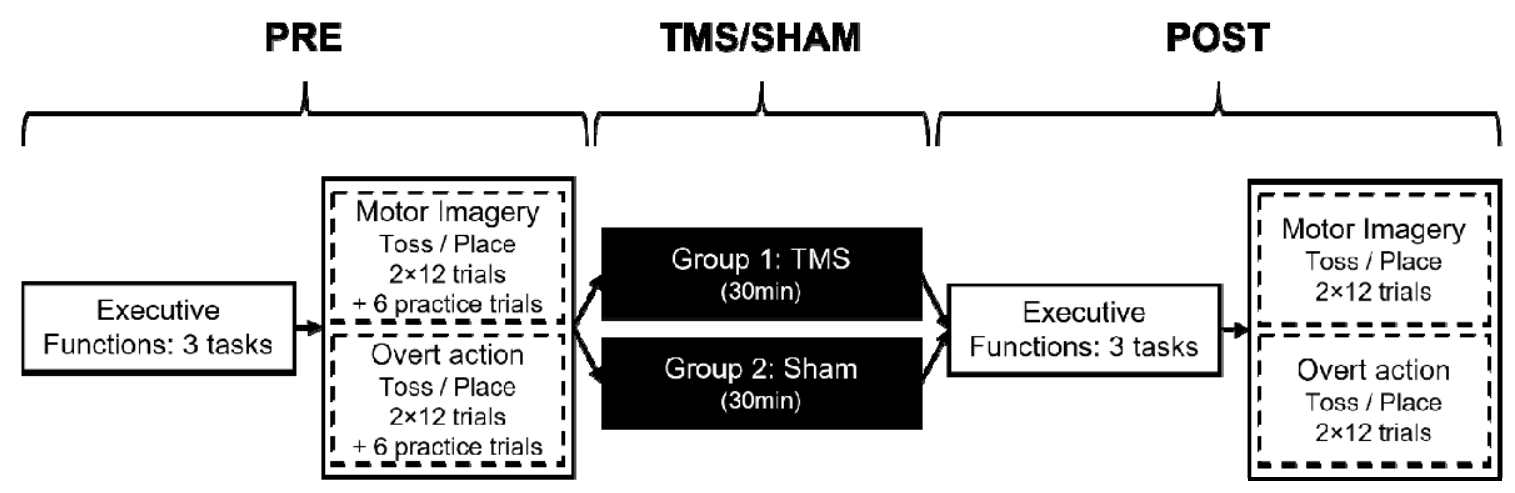

Figure 1. Experiment 1 Timeline. 


\section{Cognitive tasks}

The three cognitive tasks included: A Corsi-style Spatial span task, a Go/Nogo task, and a Switching task. Instructions were displayed on the screen prior to each task (monitor size: $47.5 \times 30 \mathrm{~cm}$, resolution: $1680 \times 1050$ px). All tasks were implemented on PsychoPy3 (v3.1.5; [75]) adapted from the Psych Toolkit website (https://www.psytoolkit.org/).

Spatial span. The Spatial span task measured visuospatial working memory performance using a computerized version of the classic Corsi-block test [76]. The layout consisted of nine squares (size: $22 \mathrm{~mm}$ ) displayed in an uneven pattern on the computer screen. At trial onset, individual squares were lightened at random (duration $=500 \mathrm{~ms}$, inter-stimuli interval $=250 \mathrm{~ms}$ ). Participants had to reproduce the full sequence by clicking on the squares with the mouse, and then clicking on a 'Done' button. Performance was not timed. The first sequence consisted of one square, with a square added to each ensuing trial following a sequence done correctly, and a square removed when participants failed to reproduce the sequence, up to a maximum of nine and minimum of one square. Different series of squares were used on each trial. Feedback was given on the computer screen for $2 \mathrm{sec}$ at the end of each trial. There were four practice trials followed by 16 analyzed trials.

Go/NoGo. The Go/NoGo task is designed to measure response inhibition [77]. It consisted of 12 practice trials, and 548 analyzed trials, with random stimulus presentations (476 Go and 84 NoGo stimuli, i.e. $85 \%$ and $15 \%$, respectively,) with $600 \mathrm{~ms}$ durations and random inter-trial intervals between 200ms and 600ms - these parameters followed the guidelines from Wessel [77] to increase the likelihood of prepotent motor responses. Participants had to press the space bar with their right index finger whenever a Go stimulus would appear ("GO - Press the space bar" written in black on a green oval) but refrain for NoGo stimulus ("NOGO - Press nothing" written in black on a red oval). A correct response for a Go trial was defined as a keypress within $600 \mathrm{~ms}$, whereas for a NoGo trial, it was defined by an absence of keypress within this time window. An incorrect response for a Go trial was an absence of a response within $600 \mathrm{~ms}$, whereas for a NoGo trial, an incorrect response occurred when the participant pressed a key within this time window. On incorrect trials, an error screen would 
appear for two seconds with a message defining the type of error ("You should not have pressed the button but you did" or "You should have pressed the button but you did not").

Switching task. The Switching task measures cognitive flexibility [78]. It consisted of responding to one item in a combination comprised of one letter and one number based on the location of the stimuli. Each stimulus appeared in a $2 \times 2$ grid at the centre of the screen. If the stimulus appeared in one of the upper quadrants, participants were required to respond to the letter. If it appeared in one of the lower quadrants, they had to respond to the number. For responses to the letter, participants were required to press the "b" key with their left index finger for consonants, and the " $\mathrm{n}$ " key with their right index finger for vowels. For numbers, participants had to press the " $b$ " key for odd values and the " $n$ " key for even. There were 8 letters (A-E-I-U-G-K-M-R) and 8 numbers (2 to 9), for 64 possible stimulus combinations. Identical numbers or letters did not appear on consecutive trials.

The task was divided into three blocks, two "control" blocks of 64 trials each, followed by a "mixed" block of 128 trials. The first 12 trials of each control block and the first 12 trials of the mixed block were considered practice and not analysed; this left a total of 104 control trials and 116 mixed trials. In the first control block, the combinations appeared only in the upper quadrants and participants always responded to the letter. In the second control block, combinations appeared only in the lower quadrants and participants always responded to the number. In both blocks, lateral positions alternated in each trial between left and right.

In the final block, trials alternated between switching and no-switching trials. Here, stimulus position rotated through every position of the quadrant in a clockwise direction. The appropriate response thus switched on every second trial between letters and numbers (e.g., when the stimulus position moved from the top right to bottom right quadrant), but did not switch on the intermingled trials (e.g., when stimulus position moved from the bottom right to bottom left quadrant). In this block then, half of the trials were "mixed, no switch", and half were "mixed, switch" trials. Participants had up to five seconds to respond. In case of an incorrect response, an error screen was displayed for three seconds with a reminder of the rules. 


\section{Action tasks}

The experimental setup for the action tasks is shown in Figure 2. Participants sat comfortably at a $50 \times$ $90 \mathrm{~cm}$ table set inside a large room. A computer keyboard, a wooden box (dimensions: $15.7 \times 42 \times 22$ $\mathrm{cm}$ ), and a small white plastic "poker chip" disc were on top of the table. The box contained a grey plastic tube (dimensions: $15 \times 4.2 \mathrm{~cm}$ ) positioned $18.5 \mathrm{~cm}$ from the participant's end of the table, and the Polhemus receiver module, positioned in the corner of the box furthest from the participant. The starting position was a yellow square sticker $(0.5 \times 0.5 \mathrm{~cm})$, positioned $52 \mathrm{~cm}$ away from the left side of the table, and $5 \mathrm{~cm}$ away from the near side. The disc was $1 \mathrm{~cm}$ thick and $4 \mathrm{~cm}$ in diameter. All other dimensions and positions were as shown in Figure 2.

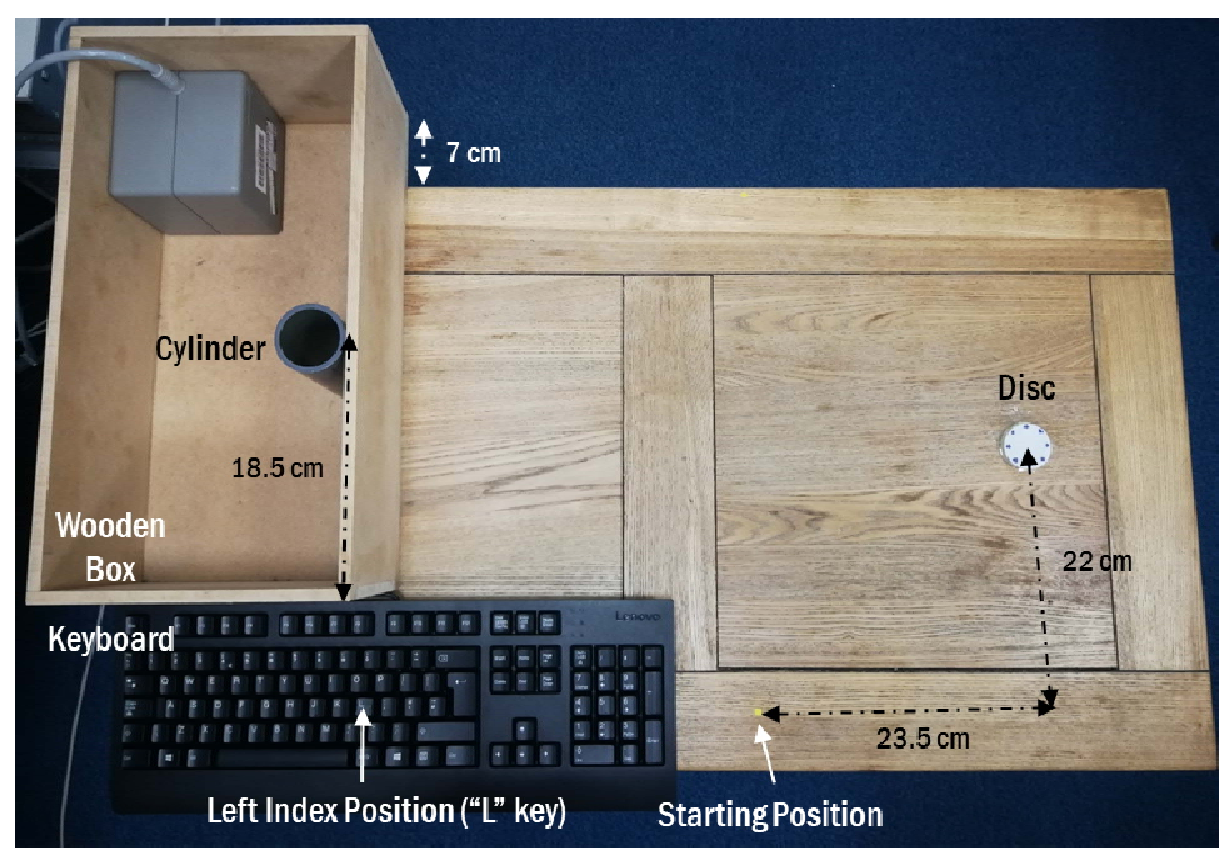

Figure 2. Experimental setup for the action tasks.

Before the start of the action tasks, two Polhemus transmitters $(7 \mathrm{~mm}$ in diameter) were placed on the participant's right thumb and index fingernails. Spatial location of these transmitters in $\mathrm{x}, \mathrm{y}, \mathrm{z}$ coordinates was recorded with a Polhemus Fastrak system (sampling rate: $120 \mathrm{~Hz}$ ). For each movement, we extracted and analyzed the data off-line. 
Participants sat with their body midline aligned with the starting position and began each trial with their right thumb and index finger held together in a comfortable "pinch" posture on the starting mark, and their left index finger resting on the "L" key. Each trial began with the experimenter triggering a tone via PC. In the overt action block, participants had to either reach for the disc and place it into the cylinder ("place" - high precision) or reach for the disc and toss it into the wooden box ("toss" - low precision). For the motor imagery block, participants had to imagine the same actions as vividly as possible, using both visual and kinaesthetic imagery of their movement experienced in the first person (i.e., to imagine seeing and feeling themselves performing the movement), with their eyes open, while keeping their right arm still. In both overt action and motor imagery sessions, participants pressed the "L" key at the beginning (or imagined beginning) of their movement, and again when releasing (or imagining releasing) the disc into the target area. During the overt action trials, the experimenter monitored participants to ensure they pressed the key at the appropriate times. In between trials, the experimenter put the disc back in place if needed and participants returned to the starting position (or remained in the same position in the motor imagery trials). Once the participant was set, the experimenter triggered the tone to begin the next trial.

\section{TMS}

Prior to applying TMS (or Sham-TMS), we determined motor threshold for each participant, defined as the minimum intensity of TMS required to result in visible twitches of the finger following stimulation of the index finger/thumb area of the right primary motor cortex. Testing stimulus intensity was set at $90 \%$ of the individual's motor threshold. Stimulation site for the right DLPFC was determined using the Beam method [79]. For the TMS group, TMS was applied over the stimulation site at $1 \mathrm{~Hz}$ for $30 \mathrm{~min}$, for a total of 1800 pulses. For the Sham group, sham TMS was administrated to the same area by inverting the coil so that the magnetic field projected away from the scalp instead of through it. Due to the length of testing, the coil tended to overheat roughly twice per session, leading to a total TMS/Sham session of approximately 40min. Mean stimulation intensity was very similar between the two groups (TMS: 58.6\%, sham: 57.8\% of stimulator output, respectively). 


\section{Data processing}

Action tasks keypress data. The first 3 trials of each task in the PRE session were considered practice. For the remaining trials, we extracted reaction time (RT) and movement time (MT) as recorded by the keypress data for both overt action and motor imagery. RT was the time between the starting tone and the first press of the key (indicating the beginning of the movement/imagery). MT was the time between first and second keypress. Any trial with RT or MT outside \pm 2 IQR (Interquartile Range) for each combination of task, session and participant was removed from the analysis $(3.8 \%$ of the total trials).

Kinematic data. Data obtained via the Polhemus was used to perform a manipulation check that participants were pressing the "L" key at times closely corresponding to their actual movement onset and offset. For the kinematic data, the beginning of the movement was set as the point at which a minimum speed of $0.05 \mathrm{~m} / \mathrm{s}$ was obtained, and three different cut-off criteria were used for the end of the movement based on velocity or grip aperture. We then compared the reaction times and movement times using these criteria to those as indexed by the keypresses in the overt action task. Reaction and movement times calculated using these cut-offs were all highly correlated with the times based on keypresses (all $r^{2} \geq 0.70$, see Supplementary Information).

Spatial span. For the sixteen experimental trials, we extracted the mean span score, computed using the formula from an adapted version of the Corsi paradigm [80]. This formula took into account both correct and incorrect responses to provide a representative score.

$$
\text { Mean span }=\frac{\sum(\text { length of correct sequence })+\sum(\text { length of incorrect sequence }-1)}{\text { number of analyzed trials }}
$$

Go/NoGo. For the 548 experimental trials, we computed the mean error rates. First, trials with RT $\leq$ $200 \mathrm{~ms}$ were removed as anticipation errors (2.3\% of total trials). Any participant with more than $10 \%$ anticipation errors in her overall performance was excluded from the experiment ( 2 participants). For 
the remaining trials, we computed the proportion of correct trials separately for Go and NoGo trials. For the analysis, the arcsine-transformed proportions were used.

Switching. For the 220experimental trials, we calculated the proportion of correct responses, then subjected these to an arcsine transformation for analysis. We then calculated the reaction time defined as the time between stimulus presentation and the participant's response. Trials without a keypress were removed ( $0.1 \%$ of the trials). RTs outside \pm 2 IQR (Interquartile Range) for each combination of task, session and participant were removed from the analysis (3.6\% of the remaining trials).

\section{Analyses}

All raw data and analyses from both experiments are available here: https://osf.io/s $82 \mathrm{v} 4 /$.

Sample size was determined a priori and was similar to that in the two previous studies using these tasks where very large effects of interference tasks on motor imagery were observed, as well as moderate effects of precision $[11,12]$. Owing to time and funding constraints, we were unable to employ more participants. Two participants who exhibited an atypical amount of anticipation errors (> $10 \%$ ) in the Go/NoGo paradigm were removed from the experiment. We analysed data for the remaining 30 participants ( 28 females).

The action tasks used a $2 \times 2 \times 2 \times 2 \times 2$ mixed design with TMS (TMS vs. SHAM) and $\operatorname{Order}(\mathrm{OA}$ First vs. MI First) as between-subjects variables, and Session (PRE vs. POST), Action (OA vs. MI), and Precision (Place vs. Toss) as within-subjects variables. We present analyses of the movement times based on keypresses. As explained in the Introduction, the critical tests revolved around the variables TMS and Action. Effects involving Precision were also expected to be present based on previous work [11]. The effects of the variables Order and Session and their interactions with other variables were not always relevant to distinguishing between the two theories under examination here, and as such, we treated these variables as exploratory. As we did not have a priori hypothesis for the reaction times, and there was no clear theoretical interpretation we could apply to any such effects, our exploratory analyses of RTs are presented separately in the Supplementary Information. 
The Spatial span and the Go/NoGo tasks used a $2 \times 2$ mixed design with TMS (TMS vs. SHAM) as a between-subjects variable and Session (PRE vs. POST) as a within-subjects variable (for the Go/NoGo task, these analyses were run separately on the Go and NoGo trials). The Switching task used a $2 \times 2 \times$ 3 mixed design with TMS as a between-subjects variable and Session and Trial Type as within-subjects variables. Trial Type had 3 levels: "control" trials in which the same rule was used on every trial (letter only and number only blocks), "mixed - no switch" trials in which the rule was the same as the previous trial, and "mixed - switch" trials in which the rule had switched from the previous trial.

Following Glover and Dixon [81], we report adjusted likelihood ratios $\left(\lambda_{\text {adj }}\right)$ of nested linear models as an index of the relative strength of competing statistical models [82]. The use of likelihood ratios avoids many of the well-known pitfalls associated with $p$-values and NHST [83-86]. In many prototypical hypothesis-testing situations, likelihood ratios bear a close relationship with $p$-values, and $\lambda_{\text {adj }}=3$ will generally correspond to $p \sim 0.05$, whereas $\lambda_{\text {adj }}=10$ will generally correspond to $p \sim 0.02$.

\section{Results}

\section{Keypress movement times}

Figure 3 shows the overall effects of the variables of Session, Precision, Action, and TMS on keypress movement times in the action tasks. We first examined effects consistent with both the MotorCognitive and Functional Equivalence views. A statistical model including effects of Session, Precision, and the Session $\times$ Precision interaction fit the data much better than did a null model $\left(\lambda_{\text {adj }}>\right.$ 1000). These results showed that movement times as indexed by the keypresses were longer in the high-precision condition (placing) as compared to the low-precision condition (tossing), were shorter in the POST session than in the PRE session, and that the decrease in MT from PRE to POST sessions was greater for placing than for tossing. All these effects were consistent with both the MotorCognitive and Functional Equivalence views. 


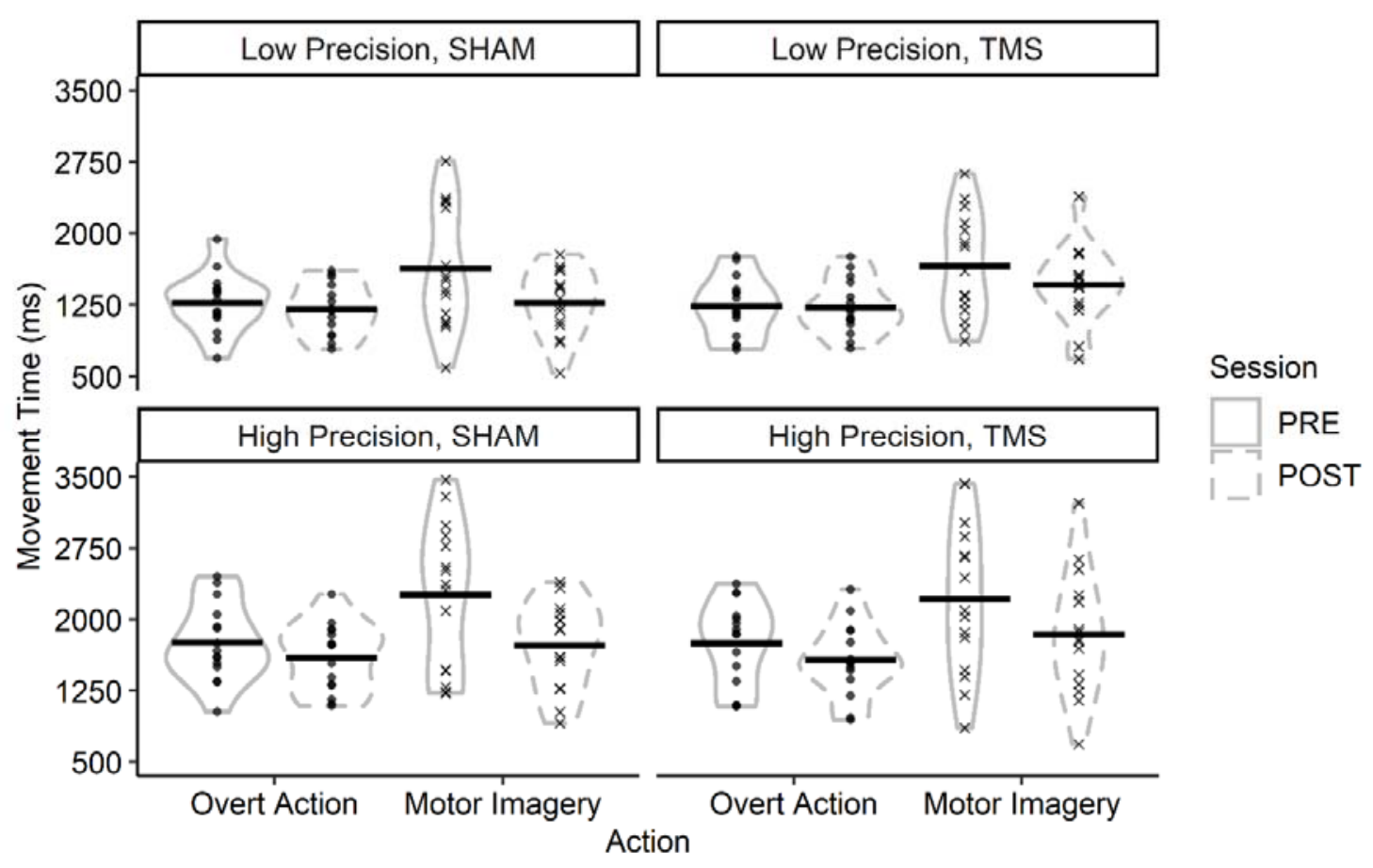

Figure 3. Effect of TMS on overt action (circle) and motor imagery (cross) movement times, for the low precision (tossing) and high precision (placing) tasks in Sham and TMS groups in the PRE (solid) and POST (dashed) sessions. Individual points represent the mean score for single participants and horizontal lines represent the group means. Enclosures represent the distribution of the presented data, with wider horizontal sections indicating a larger number of observations.

We next explored effects predicted uniquely by the Motor-Cognitive model. Adding a main effect of Action whereby movement times were longer in motor imagery than in overt action improved the fit $\left(\lambda_{\text {adj }}=552.6\right)$. Adding a Session $\times$ Action interaction further improved the fit $\left(\lambda_{\text {adj }}=90.4\right)$, indicating that movement times were overall more similar between motor imagery and overt action in the POST session than in the PRE session. The fit was improved further by adding a complex contrast in which the effect of Action was modulated by Order, but only for motor imagery, relative to a model that predicted Order effects that were independent of Action $\left(\lambda_{\text {adj }}>1000\right)$. This contrast showed that movement times for motor imagery were shorter when overt action was performed first, whereas performance in overt action was unaffected by having done motor imagery first. All these effects were consistent only with the Motor-Cognitive model. 
Most critically, the fit was further improved by adding a contrast whereby the effects of TMS were limited to motor imagery trials in the POST session $\left(\lambda_{\text {adj }}=7.10\right.$; see Figure 4$)$. This was as predicted by the Motor-Cognitive model. However, the evidence did not support the fit of a contrast whereby the effect of Precision was greater in the motor imagery than in overt action $\left(\lambda_{\text {adj }}=1.16\right)$, as also predicted by the Motor-Cognitive model and had been observed in a previous study [11].

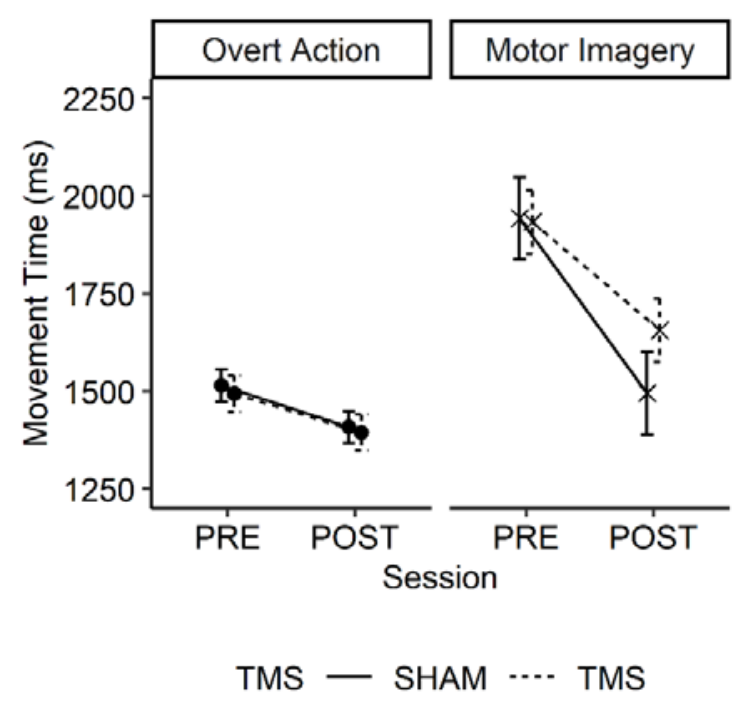

Figure 4. Effects of TMS on overt action and motor imagery keypress movement times in Sham (solid) and TMS (dashed) groups in the PRE and POST sessions. Error bars represent standard errors of the pre-post difference and are appropriate for within-subject comparisons.

Finally, to summarize the relative ability of the Motor-Cognitive and Functional Equivalence models to predict the keypress MT data, we compared a statistical model including all the factors predicted by the Motor-Cognitive model (Session, Precision, Session $\times$ Precision, Action, Session $\times$ Action, and contrasts involving interactions between Action $\times$ Order, Action $\times T M S$, and Action $\times$ Precision) to one including only the factors consistent with both theoretical views (Session, Precision, Session $\times$ Precision). The former model provided a much better fit $\left(\lambda_{\text {adj }}>1000\right)$. In other words, the data as a whole were over a thousand times more likely to occur when including those effects uniquely predicted by the Motor-Cognitive model than when including only those effects consistent with both the Motor-Cognitive and Functional Equivalence models.. 


\section{Cognitive Tasks}

Spatial span. A model assuming an effect of Session (PRE vs. POST) fit the data much better than did a null model $\left(\lambda_{\text {adj }}=268\right)$, showing that performance overall improved with practice. Adding an interaction Session $\times$ TMS improved the fit fairly modestly $\left(\lambda_{\text {adj }}=2.2\right)$, suggesting that the TMS may have led to slightly less improvement following practice relative to Sham stimulation (mean $\pm \mathrm{SD}$; Sham: $5.1 \pm 0.6$ vs. $5.7 \pm 0.5$ in POST; TMS: $5.0 \pm 0.7$ vs. $5.3 \pm 0.7$ items in POST).

Go/NoGo. Mean accuracy for the different conditions in the Go/NoGo task are shown in Table 1. For Go trials, the evidence favoured the null over a model including effects of TMS, Session, and TMS $\times$ Session $\left(\lambda_{\text {adj }}=6.51\right)$. For the NoGo trials, the best-fitting model included only an effect of Session in which accuracy decreased with practice (PRE vs. POST; $\lambda_{\text {adj }}>1000$ ). In sum, there was no evidence that TMS negatively affected performance in the Go/NoGo task.

Table 1. Accuracy in Go and NoGo trials before and after the (Sham) TMS session.

\begin{tabular}{|l|c|c|c|c|}
\hline & \multicolumn{2}{|c|}{ Mean accuracy in Go trials (\%) } & \multicolumn{2}{c|}{ Mean accuracy in NoGo trials (\%) } \\
\hline & PRE & POST & PRE & POST \\
\hline Sham & 94.7 & 95.1 & 77.1 & 68.4 \\
\hline TMS & 95.6 & 95.6 & 74.6 & 68.8 \\
\hline
\end{tabular}

Switching - Accuracy. Data from the switching task are shown in Table 2. The best-fitting model for the proportion of correct responses included only an effect of Trial Type ( $\left.\lambda_{\text {adj }}>1000\right)$, indicating reduced accuracy for the Mixed-Switch trials. Adding the main effect of TMS and its interaction with Trial Type slightly worsened the fit ( $\lambda_{\mathrm{adj}}=0.54$, or $\lambda_{\mathrm{adj}}=1.87$ in favour of the simpler model). In short, there was no evidence that TMS affected accuracy in this task.

Switching - Reaction Times. The best-fitting model included effects of Trial Type, Session, and the Trial Type $\times$ Session interaction $\left(\lambda_{\text {adj }}>1000\right)$, in which participants were overall faster in the control task, faster in the POST session, and the reduction in RT across sessions was greater in the more difficult trials (Mixed - No switch and Mixed - Switch). This model fit better than a model that also 
included effects of TMS and its interaction with session $\left(\lambda_{\text {adj }}=5.76\right)$, again suggesting TMS did not affect performance.

Table 2. Accuracy and reaction times in the task-switching.

\begin{tabular}{|c|c|c|c|c|c|c|c|c|}
\hline \multirow{2}{*}{} & \multicolumn{4}{|c|}{ Mean accuracy (\%) } & \multicolumn{3}{c|}{ Mean reaction times \pm SD (ms) } \\
\cline { 2 - 9 } & \multicolumn{2}{|c|}{ Sham } & \multicolumn{2}{c|}{ TMS } & \multicolumn{2}{c|}{ Sham } & \multicolumn{2}{c|}{ TMS } \\
\cline { 2 - 9 } & PRE & POST & PRE & POST & PRE & POST & PRE & POST \\
\hline Control & 96.3 & 93.7 & 95.4 & 95.5 & $649 \pm 118$ & $574 \pm 56$ & $631 \pm 63$ & $590 \pm 46$ \\
\hline $\begin{array}{c}\text { Mixed - } \\
\text { No Switch }\end{array}$ & 95.6 & 95.2 & 96.6 & 96.4 & $880 \pm 236$ & $722 \pm 165$ & $843 \pm 168$ & $727 \pm 111$ \\
\hline $\begin{array}{c}\text { Mixed - } \\
\text { Switch }\end{array}$ & 92.5 & 93.4 & 91.3 & 92.3 & $1175 \pm 398$ & $933 \pm 297$ & $1177 \pm 299$ & $929 \pm 195$ \\
\hline
\end{tabular}

\section{Discussion}

Keypress movement time data from the action task strongly supported the Motor-Cognitive-model over the Functional Equivalence model. Critically, TMS was associated with longer movement times in the motor imagery task only, leaving overt actions unaffected. Practice between PRE and POST improved the correspondence between the timing of motor imagery and overt action to a greater extent in the Sham Group than the TMS group, reinforcing the idea that disrupting the DLPFC disrupted the ability to perform motor imagery effectively. Of some interest, our exploratory tests of order effects found that whether overt action was performed before or after motor imagery affected movement times. This may have arisen because with physical practice a better representation of the movement becomes available to motor imagery [65].

Results of the cognitive tasks were inconsistent. TMS may have slightly disrupted performance in the spatial span task but did not appear to affect performance in the Go/NoGo or switching tasks. This could suggest many possibilities: It may be that working memory is the executive function most important to motor imagery, or that inhibition and/or set switching do not rely on the right DLPFC. Or, it may be that motor imagery shares with all these tasks a common pool of executive resources, and 
that the cognitive tasks did not use enough of these executive resources to be clearly affected by TMS. We address these possibilities in greater detail in the General Discussion.

In Experiment 2, we simplified the design and increased the sample size to focus on a smaller number of key variables. Relative to Experiment 1, we eliminated the variables Order, Session, and Precision, and added the variable Calc [for calculation]. This variable was introduced to impose an interfering demand on executive resources, and has previously been shown to have a large impact on the timing of motor imagery with only a minimal effect on overt action $[11,12]$. The pre-registered methods, hypotheses, and analysis plan of Experiment 2 can be viewed here: https://osf.io/geqx2.

Once again, the two theories make contrasting predictions. According to the Motor-Cognitive model, only motor imagery ought to be affected by the Action, TMS and Calc variables, and in any interactions between Action and either or both of the other two variables. Further, the slowing of movement times by TMS should be greater when the calculation task is performed concurrently with motor imagery, evident in a three-way interaction. Conversely, the Functional Equivalence model predicts no main effect of Action, nor any interactions between Action and either of the TMS or Calc variables, nor a three-way $T M S \times$ Action $\times$ Calc interaction.

\section{Experiment 2}

\section{Methods}

\section{Participants}

We enrolled 64 new participants (58 females, age range 18-55 years old) from the Royal Holloway Department of Psychology undergraduate research participation pool and research staff. Two participants were replaced due to technical difficulties. Participants were randomly assigned to receive either TMS or Sham-TMS. Participants in each of these groups were further randomly assigned to take part in either the motor imagery or the overt action condition. Inclusion criteria were the same as in 
Experiment 1. Due to Covid-19 safety precautions, the last 25 participants performed all tasks with a face mask and surgical gloves on. None of the participants reported any additional difficulty in executing the tasks with these safety measures. These latter participants received a $£ 15$ retail voucher instead of credits.

\section{Tasks and setup}

The cognitive tasks, application of TMS/Sham, and the action task setup were identical to Experiment 1. Mean intensity stimulation was very similar between the TMS and Sham groups (TMS: 57.0\%, Sham: $56.8 \%$ of stimulator intensity, respectively).

\section{Procedure}

Participants first received TMS (or sham TMS) at a rate of $1 \mathrm{~Hz}$ for 30 minutes (1800 pulses) over the right dorsolateral prefrontal cortex. Immediately after TMS/Sham stimulation, they comfortably sat in front of a computer to perform the three cognitive tasks, following the same order as in Experiment 1: Spatial span, Go/NoGo, and Switching. Following this, participants performed one of the motor tasks, either motor imagery or overt action. Each participant performed two blocks of either the overt action or motor imagery version of the grasping and placing task from Experiment. One of the two blocks for each participant included the calculation task, the other was a control, counterbalanced across participants using an ABBA design.

As in Experiment 1, participants indicated the beginning and end of each movement (or its imagined counterpart) by pressing the "L" key on the keyboard. In the calculation block, just prior to the onset of the trial, the experimenter read aloud a pre-determined number between 51-99. At the sound of the tone, participants had to simultaneously count backwards out loud by threes while performing either the overt or imagined reaching and placing movement. The numbers were pseudo-randomly determined so that the same multiples of three could not occur in successive trials (as might occur if, e.g., the numbers 63 and 60 were given on two adjacent trials). Verbal responses were recorded by the experimenter for later analysis. 


\section{Data processing and statistics}

Extracted variables, outlier detection and general data processing was as in Experiment 1, and are described in the preregistration of Experiment 2 (https://osf.io/geqx2). One participant exhibited an atypical amount of anticipation errors in the Go/NoGo task (> 10\%); this participant was excluded from all analyses, which were conducted on the remaining 63 participants (57 females). The action tasks were analysed using a $2 \times 2 \times 2$ mixed design with TMS (TMS/Sham) and Action (overt action/motor imagery) as between-subjects variables, and Calc (calculation/control) as a withinsubjects variable. As a control, we also conducted an analysis including a fourth variable, TimelineCovid, which separated participants based on whether or not they had to follow safety measures such as wearing a mask and gloves. As this analysis provided strong evidence that the TimelineCovid variable did not modulate any of the other effects (see Supplementary Information), we excluded this variable from further analyses.

The number of errors on the Calculation task was computed and entered into a $2 \times 2$ mixed design with Group (TMS/Sham) and Action (overt action/motor imagery) as between-subjects variables. We had originally planned on investigating the average number of responses for the calculation task (in the pre-registration) but later realized it would not be informative as a longer trial would allow more responses than a shorter trial, so instead we computed a "time per response". This was indexed as the total number of responses given by a participant for each condition divided by the sum of movement times for each condition.

The Spatial span and the Go/NoGo tasks had a between-subjects design with TMS (TMS or SHAM) as the between-subjects variable, and the Switching task added the within-subjects variable Trial Type (control, mixed-no switch, and mixed-switch). As before, we reported the fit of nested linear models using adjusted likelihood ratios, $\lambda_{\text {adj. }}$. 


\section{Results}

\section{Keypress movement times}

Figure 5 shows the effects of the Action, Calc, and Group variables on keypress movement times in the overt and imagined reaching and grasping task. As in Experiment 1, we focussed our tests on the competing predictions of the Motor-Cognitive and Functional Equivalence models. Although the tests described here fulfilled this goal, note that the present analysis diverted somewhat from the preregistered plan which had been based on predicted effect sizes, as the formulation of said plan turned out to include miscalculations which we only noticed after we had collected the data. In lieu of the preregistered analyses, the analyses undertaken were the same conventional and statistically sound tests of nested linear models used in Experiment 1.

We again began by testing statistical models consistent with both theoretical views. A model including a main effect of TMS whereby movement times were longer in the TMS group than in the Sham group fit the data somewhat better than a null model $\left(\lambda_{\text {adj }}=3.66\right)$. There was also strong evidence for both a main effect of Calc and a TMS $\times$ Calc interaction wherein movement times were longer in the calculation condition, particularly for the TMS group $\left(\lambda_{\text {adj }}>1000\right)$. These effects strongly suggested that TMS interfered with subsequent performance of the calculation task, and were consistent with both the Motor-Cognitive and Functional Equivalence views.

We next examined effects predicted solely by the Motor-Cognitive model. Including the main effect of Action and the Action $\times$ TMS interaction, improved the fit greatly $\left(\lambda_{\text {adj }}>1000\right)$. This provided strong evidence that movement times were longer overall in the motor imagery group than in the overt action group, and longer still in the TMS group in the motor imagery condition compared to the overt action condition. Adding in an Action $\times$ Calc interaction, wherein movement times were longer in the calculation condition than in the control condition, and with this effect being greater in the motor imagery condition, improved the fit even more $\left(\lambda_{\text {adj }}=226\right)$. However, there was little evidence to support adding the three-way interaction Action $\times$ Calc $\times T M S$, also predicted solely by the MotorCognitive Model; including this interaction neither improved nor weakened the fit $\left(\lambda_{\text {adj }}=1.03\right)$. 
Finally, we again compared a statistical model including all the effects uniquely predicted by the Motor-Cognitive model (Action, Action $\times$ TMS, Action $\times$ Calc, and Action $\times$ Calc $\times T M S$ ) to one including only those effects consistent with both Motor-Cognitive and Functional Equivalence views (TMS, Calc, TMS $\times$ Calc). This analysis showed strong support for including those effects unique to the Motor-Cognitive view ( $\left.\lambda_{\text {adj }}>1000\right)$. In short, the data were more than 1000 times as likely assuming the effects predicted solely by the Motor-Cognitive model than assuming only those effects predicted by the Functional Equivalence view.

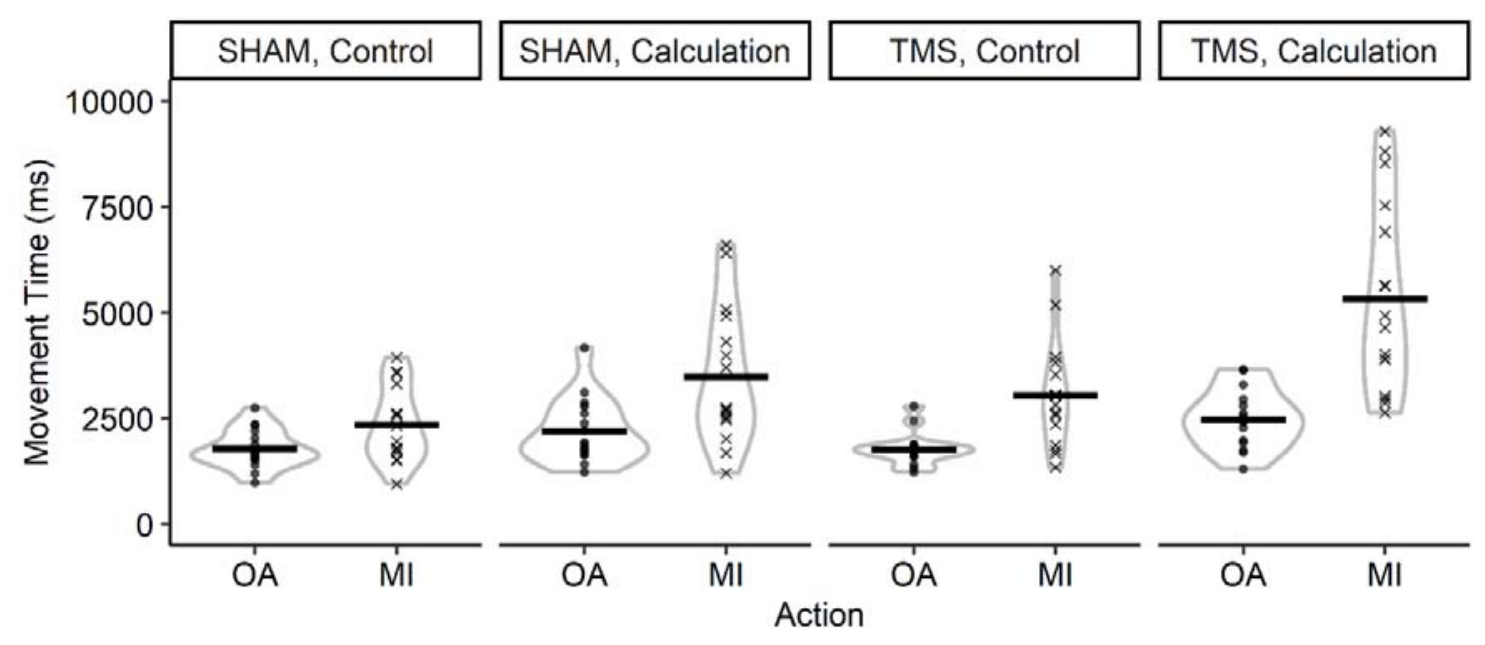

Figure 5. Effect of Sham vs. TMS on overt action (OA) and motor imagery (MI) movement times, for the control and the calculation tasks. Horizontal lines represent the group means, individual points represent the means for single participants. Enclosures represent the distribution of the presented data, with wider horizontal sections indicating a larger number of observations.

\section{Calculation Task}

Accuracy and time-per-response performance for the calculation task are summarised in Table 3. For the mean time per response, the best-fitting model included main effects of both TMS and Action $\left(\lambda_{\text {adj }}\right.$ $=102.1)$. This indicated that participants took more time to calculate a number after TMS stimulation compared to Sham, and during the motor imagery compared to overt action condition, confirming that both TMS and motor imagery negatively affected performance of the calculation task. However, for 
error rates a null model fit the data better than a full model incorporating effects of $T M S_{s}$ Action, and $T M S \times$ Action $\left(\lambda_{\text {adj }}=9.2\right)$, suggesting none of these variables affected accuracy. The effects of TMS and Action on time per response, coupled with the lack of similar effects on accuracy, indicated that the former did not result from participants employing a speed-accuracy trade-off.

Table 3. Calculation task performance.

\begin{tabular}{|c|c|c|c|c|}
\hline \multirow{2}{*}{} & \multicolumn{2}{|c|}{ Mean Time Per Number \pm SD $(\mathbf{m s})$} & \multicolumn{2}{l|}{ Error rate (\%) } \\
\cline { 2 - 5 } & MI & OA & MI & OA \\
\hline Sham & $1780 \pm 530$ & $1486 \pm 658$ & 4.56 & 7.56 \\
\hline TMS & $2741 \pm 1343$ & $1831 \pm 993$ & 6.98 & 7.54 \\
\hline
\end{tabular}

\section{Cognitive Tasks}

Spatial span. Spatial span score in Experiment 2 was explained slightly better by a null model that assumed no difference between TMS (5.3 \pm 0.8 items) and Sham (5.2 \pm 0.7 items $)$ groups than by a model including an effect of TMS $\left(\lambda_{\text {adj }}=2.42\right)$. Thus, unlike in Experiment 1, the evidence modestly suggested TMS did not affect performance of this task.

Go/NoGo. Accuracy performance here was also similar between TMS and Sham groups in the Go trials, with a null model assuming no difference between TMS (95.1\%) and Sham (95.5\%) again providing a slightly better account of the data than a model assuming an effect of $T M S\left(\lambda_{\text {adj }}=2.38\right)$. For the NoGo trials, the evidence did not distinguish between the null and a model including the effect of TMS ( $\lambda_{\text {adj }}=1.08$ in favour of the null; TMS: $77.3 \%$; Sham: 73.6\%).

Switching - Accuracy. Table 4 shows mean accuracy and reaction times for the switching task. The best fitting-model included a large effect of Trial Type $\left(\lambda_{\text {adj }}>1000\right)$ whereby accuracy was lower in the mixed-switch trials than in the control or mixed-no switch trials. However, adding a main effect of TMS and an interaction between TMS and Trial Type did not improve the fit at all $\left(\lambda_{\text {adj }}=1.0\right)$, suggesting that TMS did not affect accuracy. 
Switching - Reaction Times. The best fitting-model included a large effect of Trial Type $\left(\lambda_{\text {adj }}>1000\right)$ whereby reaction times were higher the more difficult the task was. Adding in a contrast whereby TMS slowed reaction times in the mixed-no switch and mixed-switch trials, but not in the control trials, did not notably improve the fit $\left(\lambda_{\text {adj }}=1.26\right)$.

Table 4. Accuracy and reaction times in the task-switching paradigm.

\begin{tabular}{|c|c|c|c|c|}
\hline & \multicolumn{2}{|c|}{ Mean accuracy (\%) } & \multicolumn{2}{c|}{ Mean reaction times \pm SD (ms) } \\
\hline & Sham & TMS & Sham & TMS \\
\hline Control & 95.3 & 96.2 & $661 \pm 86$ & $683 \pm 88$ \\
\hline Mixed - No switch & 97.6 & 96.6 & $811 \pm 149$ & $917 \pm 188$ \\
\hline Mixed - Switch & 93.6 & 92.7 & $1152 \pm 261$ & $1223 \pm 292$ \\
\hline
\end{tabular}

\section{Discussion}

The results of Experiment 2 provided further support for the Motor-Cognitive model over the Functional Equivalence view. Critically, TMS had much greater effects on keypress movement times for motor imagery than for overt action. This suggested that disrupting DLPFC activity with TMS interfered with the timing of motor imagery, but left overt actions relatively unaffected. Similarly, in line with previous studies $[11,12]$, the calculation task also had a slowing effect on motor imagery but not on overt action. Overall, a statistical model that incorporated all the effects predicted solely by the Motor-Cognitive model performed much better than one including only those effects predicted by both the Motor-Cognitive and Functional Equivalence views.

TMS also had large effects on performance of the calculation task. Specifically, there was strong evidence that TMS lengthened the amount of time required to generate each number in the calculation task, relative to sham stimulation. Evidence for effects of TMS on the other three cognitive tasks, however, was inconclusive. Unlike in Experiment 1, TMS did not appear to affect performance of the spatial span task, and for the Go/NoGo and switching tasks, there was again no evidence that TMS impacted their performance. 
TMS Over Dorsolateral 25

\section{General Discussion}

Support for the Motor-Cognitive Model

The results of the two experiments here strongly support the idea, inherent in the Motor-Cognitive model, that the DLPFC plays an important role in motor imagery. When DLPFC activity was disrupted by TMS, keypress movement times became longer in motor imagery tasks relative to sham stimulation, but the same effect was not observed in the overt action tasks. These findings are consistent with several studies using fMRI showing increased DLPFC activation during motor imagery [18,29-38]. However, they contradict a core principle of the Functional Equivalence view, which holds that motor imagery utilizes the same internal processes as overt actions, and thus both motor imagery and overt action should be equally affected (or unaffected) by disruption of the DLPFC.

The present study also replicated many of the main findings of Glover and colleagues [11,12]. First, motor imagery of the reaching, grasping and placing/tossing actions consistently took longer than the corresponding overt actions, independent of TMS or other manipulations. Second, adding an interference task greatly lengthened the time required to complete the motor imagery task, but had much smaller effects on overt actions. Replication of these findings provide further support to the Motor-Cognitive model over the principle of Functional Equivalence.

\section{TMS and Cognitive Tasks}

The effects of TMS on the calculation task and the three other cognitive tasks were inconsistent, however. These are important results to try to disentangle, as the logic of the design was that TMS over DLPFC would disrupt executive functions, which in principle ought to have affected all tasks thought to use these functions. Although TMS had a very large and unequivocal effect on the time required to give responses in the calculation task of Experiment 2, its effects on the other cognitive tasks were not generally evident; only weak evidence for an effect of TMS on the Spatial span task of Experiment 1 was observed, and this was not replicated for the same task in Experiment 2. 
There are several possible explanations for why this pattern of results may have arisen. Each of the cognitive tasks we employed taps into different types of executive functions, and in fact one reason why we employed a wide range of tasks was to explore which of these functions might ally most with the functions used to support motor imagery. For example, spatial span performance is largely dependent on spatial working memory, which could presumably also be important in motor imagery. However, working memory is a largely distributed process that relies not just on the right DLPFC $[87,88]$, but a large network of areas, including the left DLFPC $[21,89]$, the ventral prefrontal cortex [90], the precentral sulcus [91] and the posterior parietal lobes [92-94]. Thus, disrupting the right DLPFC alone may have been insufficient to reliably affect spatial working memory.

Performance in the Go/NoGo task depends on response inhibition, a process that has been argued to play an important role in motor imagery $[95,96]$. On this account, motor imagery must inhibit the preplanned motor response and instead rehearse it mentally. Although we agree that there must be some element of inhibition in motor imagery, we did not find effects of TMS on Go/NoGo performance. It may be that the inhibitory element of the Go/NoGo task does not depend on the right DLPFC; rather it may rely on other prefrontal regions such as the left DLPFC, or more ventral regions of the prefrontal cortex $[97,98]$.

Switching task performance has been attributed to at least two not mutually exclusive processes: working memory to select and maintain tasks sets, and a process to reduce interference from stimulus ambiguity [99,100]. These functions, activating frontal areas in the brain [101] require cognitive flexibility and executive resources, and thus could arguably be matched with processes supporting motor imagery, and so expected to be impaired by TMS over the right DLPFC. Yet here, no such effects were observed.

Given the generally absent effects of TMS on these three cognitive tasks, it may seem surprising that it did have large effects on the calculation task. As an internally-generated response, the calculation task may require a greater amount of executive resources than the other three cognitive tasks [102-104]. It also appears to be introspectively quite demanding of mental resources. Perhaps not coincidentally, motor imagery is also internally-generated and is also introspectively a fairly demanding mental task. 
Further to this, performing arithmetic heavily activates the right DLPFC [89]. Each of these factors may have contributed to the TMS affecting performance of the calculation task and motor imagery, but not the other three cognitive tasks. In short then, the three cognitive tasks we used to measure the effects of TMS over the DLPFC on executive functions may have been focussed fairly specifically on individual executive functions such as working memory, inhibition, and task switching, and less reliant on the general pool of executive resources. Cognitive tasks requiring internally-generated responses might indeed be more sensitive to TMS over the DLPFC, tasks such as the calculation task used here, word generation, generating novel uses of a common object, or reciting the months of the calendar in reverse order.

\section{Practical Applications of the Motor-Cognitive Model}

Given the support that has been found for the Motor Cognitive model here and in other recent work $[11,12]$, it is worth exploring its potential applications in training and rehabilitation. Current techniques rely heavily on the notion of functional equivalence $[105,106]$.But if the Motor Cognitive model is a nearer approximation of the organisation of motor imagery, this could have important implications for the treatment of many motor and cognitive disorders, as well as the typical or atypical development of motor skills.

In training and rehabilitation, for example, the Motor-Cognitive model would suggest that the best outcomes for motor imagery training would be those for which a strong motor representation of the action is already present. This would result in a higher fidelity between the initial formulation of the motor image and its corresponding overt action during the planning stage, resulting in a lower reliance of motor imagery on inefficient (in terms of resources) and low fidelity (in terms of macroscopic and microscopic movement elements) executive functions to elaborate the motor image [11]. Consistent with this view, completely novel movements for which no internal motor representation exists cannot be learned with motor imagery alone [6,107]. For novel or otherwise poorly represented actions, a better approach might be to use action observation to help build internal representations $[108,109]$, which can then be strengthened with motor imagery. 
A common motor disorder that may respond positively to incorporating the Motor-Cognitive model into its treatment is Developmental Coordination Disorder (DCD), in which persons experience impaired motor skills from an early age, negatively impacting academic and life achievements [110]. Consistent with the Motor-Cognitive model, these individuals also present with reduced motor imagery abilities and deficits in executive functions [111,112]. Treatment of DCD may potentially benefit from incorporating the tenets of the Motor-Cognitive model as suggested above - i.e., the first step towards improvement of motor function ought to involve the building and strengthening of internal motor representations, either through overt practice or action observation, which may then be supplemented with motor imagery training.

Patients with damage to the DLPFC also report poor motor imagery abilities [113]. A prediction of the Motor-Cognitive model is that these deficits should be most evident for imagined actions which rely most heavily on executive resources, dependent on the DLPFC. If this can be confirmed empirically, then rehabilitation efforts for patients with DLPFC involvement might follow the same guidance as above, perhaps even more strenuously, as those with DLPFC damage should be less able to mentally elaborate a motor image for which only a poor internal representation exists.

\section{Conclusion}

The present study demonstrated an important role of the dorsolateral prefrontal cortex in motor imagery. When TMS was used to disrupt the right DLPFC, participants took longer to perform motor imagery compared to a sham-TMS condition. TMS had a similar effect on a calculation task designed to utilise a general pool of executive resources. TMS did not, however, reliably affect cognitive tasks more focussed on specific executive functions. These results support the idea, inherent in the MotorCognitive model, that the DLPFC plays an important role in motor imagery, and provide further evidence against the notion of a functional equivalence between motor imagery and overt actions. This may have important implications for the use of motor imagery in training and rehabilitation. 


\section{Acknowledgements}

This work was supported by the BIAL foundation (Grant 193/18). We thank Jai Patel, Emily Durber,

Mollie Barker and Vykintas Maziukas for their help with data acquisition.

\section{Competing interests}

There are no conflicts of interest.

\section{Data availability statement}

All data, code and material used in this study are available on the website of the Open Science Framework (OSF) at the following link: https://osf.io/s82v4/

\section{References}

[1] A. Guillot, C. Collet, Construction of the Motor Imagery Integrative Model in Sport: a review and theoretical investigation of motor imagery use, Int. Rev. Sport Exerc. Psychol. 1 (2008) 3144. https://doi.org/10.1080/17509840701823139.

[2] C. Ruffino, C. Papaxanthis, F. Lebon, Neural plasticity during motor learning with motor imagery practice: Review and perspectives, Neuroscience. 341 (2017) 61-78. https://doi.org/10.1016/j.neuroscience.2016.11.023.

[3] U.M. Bello, S.J. Winser, C.C.H. Chan, Role of kinaesthetic motor imagery in mirror-induced visual illusion as intervention in post-stroke rehabilitation, Rev. Neurosci. 31 (2020) 659-674. https://doi.org/10.1515/revneuro-2019-0106.

[4] P.L. Jackson, M.F. Lafleur, F. Malouin, C. Richards, J. Doyon, Potential role of mental practice using motor imagery in neurologic rehabilitation, Arch. Phys. Med. Rehabil. 82 (2001) 11331141. https://doi.org/10.1053/apmr.2001.24286.

[5] F. Malouin, C.L. Richards, Mental practice for relearning locomotor skills, Phys. Ther. 90 (2010) 240-251. https://doi.org/10.2522/ptj.20090029.

[6] T. Mulder, Motor imagery and action observation: cognitive tools for rehabilitation, J. Neural Transm. (Vienna). 114 (2007) 1265-1278. https://doi.org/10.1007/s00702-007-0763-z.

[7] N. Sharma, V.M. Pomeroy, J.-C. Baron, Motor imagery: a backdoor to the motor system after stroke?, Stroke. 37 (2006) 1941-1952. https://doi.org/10.1161/01.STR.0000226902.43357.fc. 
[8] A. Moran, A. Guillot, T. MacIntyre, C. Collet, Re-imagining motor imagery: building bridges between cognitive neuroscience and sport psychology, Br. J. Psychol. 103 (2012) 224-247. https://doi.org/10.1111/j.2044-8295.2011.02068.x.

[9] A. Dietrich, Imaging the imagination: the trouble with motor imagery, Methods. 45 (2008) 319-324. https://doi.org/10.1016/j.ymeth.2008.04.004.

[10] H. O'Shea, A. Moran, Does motor simulation theory explain the cognitive mechanisms underlying motor Imagery? A critical review, Front. Hum. Neurosci. 11 (2017) 72. https://doi.org/10.3389/fnhum.2017.00072.

[11] S. Glover, M. Baran, The motor-cognitive model of motor imagery: evidence from timing errors in simulated reaching and grasping, J. Exp. Psychol. Hum. Percept. Perform. 43 (2017) 1359-1375. https://doi.org/10.1037/xhp0000389.

[12] S. Glover, E. Bibby, E. Tuomi, Executive functions in motor imagery: support for the motorcognitive model over the functional equivalence model, Exp. Brain Res. 238 (2020) 931-944. https://doi.org/10.1007/s00221-020-05756-4.

[13] M. Jeannerod, V. Frak, Mental imaging of motor activity in humans, Curr. Opin. Neurobiol. 9 (1999) 735-739. https://doi.org/10.1016/s0959-4388(99)00038-0.

[14] M. Jeannerod, Mental imagery in the motor context, Neuropsychologia. 33 (1995) 1419-1432. https://doi. org/10.1016/0028-3932(95)00073-C.

[15] M. Jeannerod, J. Decety, Mental motor imagery: a window into the representational stages of action, Curr. Opin. Neurobiol. 5 (1995) 727-732. https://doi.org/10.1016/09594388(95)80099-9.

[16] S. Glover, Separate visual representations in the planning and control of action, Behav. Brain Sci. 27 (2004) 3-24. https://doi.org/10.1017/S0140525X04000020.

[17] S. Glover, P. Dixon, Context and vision effects on real and imagined actions: support for the common representation hypothesis of motor imagery, J. Exp. Psychol. Hum. Percept. Perform. 39 (2013) 1352-1364. https://doi.org/10.1037/a0031276.

[18] M.-S. Vry, D. Saur, M. Rijntjes, R. Umarova, P. Kellmeyer, S. Schnell, V. Glauche, F. Hamzei, C. Weiller, Ventral and dorsal fiber systems for imagined and executed movement, Exp. Brain Res. 219 (2012) 203-216. https://doi.org/10.1007/s00221-012-3079-7.

[19] R. Shadmehr, J.W. Krakauer, A computational neuroanatomy for motor control, Exp. Brain Res. 185 (2008) 359-381. https://doi.org/10.1007/s00221-008-1280-5.

[20] D.M. Wolpert, R.C. Miall, M. Kawato, Internal models in the cerebellum, Trends Cogn. Sci. (Regul. Ed.). 2 (1998) 338-347. https://doi.org/10.1016/S1364-6613(98)01221-2.

[21] Y. Bagherzadeh, A. Khorrami, M.R. Zarrindast, S.V. Shariat, D. Pantazis, Repetitive transcranial magnetic stimulation of the dorsolateral prefrontal cortex enhances working memory, Exp. Brain Res. 234 (2016) 1807-1818. https://doi.org/10.1007/s00221-016-4580-1.

[22] G. Blasi, T.E. Goldberg, T. Weickert, S. Das, P. Kohn, B. Zoltick, A. Bertolino, J.H. Callicott, D.R. Weinberger, V.S. Mattay, Brain regions underlying response inhibition and interference monitoring and suppression, Eur. J. Neurosci. 23 (2006) 1658-1664. https://doi.org/10.1111/j.1460-9568.2006.04680.x.

[23] M. D'Esposito, B.R. Postle, B. Rypma, Prefrontal cortical contributions to working memory: evidence from event-related fMRI studies, Exp. Brain Res. 133 (2000) 3-11. https://doi.org/10.1007/s002210000395.

[24] S.J. Gilbert, P.W. Burgess, Executive function, Curr. Biol. 18 (2008) R110-114. https://doi.org/10.1016/j.cub.2007.12.014.

[25] R.B. Mars, M.J. Grol, Dorsolateral prefrontal cortex, working memory, and prospective coding for action, J. Neurosci. 27 (2007) 1801-1802. https://doi.org/10.1523/jneurosci.5344-06.2007.

[26] T.A. Niendam, A.R. Laird, K.L. Ray, Y.M. Dean, D.C. Glahn, C.S. Carter, Meta-analytic evidence for a superordinate cognitive control network subserving diverse executive functions, Cogn. Affect. Behav. Neurosci. 12 (2012) 241-268. https://doi.org/10.3758/s13415-011-0083-5. 
[27] P. Yuan, N. Raz, Prefrontal cortex and executive functions in healthy adults: a meta-analysis of structural neuroimaging studies, Neurosci. Biobehav. Rev. 42 (2014) 180-192. https://doi.org/10.1016/j.neubiorev.2014.02.005.

[28] T.P. Zanto, M.T. Rubens, A. Thangavel, A. Gazzaley, Causal role of the prefrontal cortex in topdown modulation of visual processing and working memory, Nat. Neurosci. 14 (2011) 656661. https://doi.org/10.1038/nn.2773.

[29] L. Dodakian, J.C. Stewart, S.C. Cramer, Motor imagery during movement activates the brain more than movement alone after stroke: a pilot study, J. Rehabil. Med. 46 (2014) 843-848. https://doi.org/10.2340/16501977-1844.

[30] S. Gardini, A. Venneri, W.J. McGeown, C. Toraci, L. Nocetti, C.A. Porro, P. Caffarra, Brain activation patterns characterizing different phases of motor action: execution, choice and ideation, Brain Topogr. 29 (2016) 679-692. https://doi.org/10.1007/s10548-016-0491-5.

[31] E. Gerardin, A. Sirigu, S. Lehéricy, J.B. Poline, B. Gaymard, C. Marsault, Y. Agid, D. Le Bihan, Partially overlapping neural networks for real and imagined hand movements, Cereb. Cortex. 10 (2000) 1093-1104. https://doi.org/10.1093/cercor/10.11.1093.

[32] A. Guillot, C. Collet, V.A. Nguyen, F. Malouin, C. Richards, J. Doyon, Functional neuroanatomical networks associated with expertise in motor imagery, Neuroimage. 41 (2008) 1471-1483. https://doi.org/10.1016/j.neuroimage.2008.03.042.

[33] A. Guillot, C. Collet, V.A. Nguyen, F. Malouin, C. Richards, J. Doyon, Brain activity during visual versus kinesthetic imagery: an fMRI study, Hum. Brain Mapp. 30 (2009) 2157-2172. https://doi. org/10.1002/hbm.20658.

[34] M. Lotze, G. Scheler, H.-R.M. Tan, C. Braun, N. Birbaumer, The musician's brain: functional imaging of amateurs and professionals during performance and imagery, Neuroimage. 20 (2003) 1817-1829. https://doi.org/10.1016/j.neuroimage.2003.07.018.

[35] K.L. Macuga, S.H. Frey, Differential contributions of the superior and inferior parietal cortex to feedback versus feedforward control of tools, Neuroimage. 92 (2014) 36-45. https://doi.org/10.1016/j.neuroimage.2014.01.024.

[36] K.M. Stephan, G.R. Fink, R.E. Passingham, D. Silbersweig, A.O. Ceballos-Baumann, C.D. Frith, R.S. Frackowiak, Functional anatomy of the mental representation of upper extremity movements in healthy subjects, J. Neurophysiol. 73 (1995) 373-386. https://doi.org/10.1152/jn.1995.73.1.373.

[37] A. Tacchino, C. Saiote, G. Brichetto, G. Bommarito, L. Roccatagliata, C. Cordano, M.A. Battaglia, G.L. Mancardi, M. Inglese, Motor imagery as a function of disease severity in multiple sclerosis: an fMRI study, Front. Hum. Neurosci. 11 (2017) 628. https://doi.org/10.3389/fnhum.2017.00628.

[38] L. Zapparoli, P. Invernizzi, M. Gandola, M. Verardi, M. Berlingeri, M. Sberna, A. De Santis, A. Zerbi, G. Banfi, G. Bottini, E. Paulesu, Mental images across the adult lifespan: a behavioural and fMRI investigation of motor execution and motor imagery, Exp. Brain Res. 224 (2013) 519-540. https://doi.org/10.1007/s00221-012-3331-1.

[39] C.-J. Olsson, L. Nyberg, Brain simulation of action may be grounded in physical experience, Neurocase. 17 (2011) 501-505. https://doi.org/10.1080/13554794.2010.547504.

[40] M. Moriya, K. Sakatani, Effects of motor imagery on cognitive function and prefrontal cortex activity in normal adults evaluated by NIRS, Adv. Exp. Med. Biol. 977 (2017) 227-231. https://doi.org/10.1007/978-3-319-55231-6_31.

[41] Y.K. Kim, E. Park, A. Lee, C.-H. Im, Y.-H. Kim, Changes in network connectivity during motor imagery and execution, PLoS One. 13 (2018) e0190715. https://doi.org/10.1371/journal.pone.0190715.

[42] N. Mizuguchi, H. Nakata, T. Hayashi, M. Sakamoto, T. Muraoka, Y. Uchida, K. Kanosue, Brain activity during motor imagery of an action with an object: a functional magnetic resonance imaging study, Neurosci. Res. 76 (2013) 150-155.

https://doi.org/10.1016/j.neures.2013.03.012. 
[43] M. Lee, J.-G. Yoon, S.-W. Lee, Predicting motor imagery performance from resting-state EEG using dynamic causal modeling, Front. Hum. Neurosci. 14 (2020) 321.

https://doi.org/10.3389/fnhum.2020.00321.

[44] T. Zhang, T. Liu, F. Li, M. Li, D. Liu, R. Zhang, H. He, P. Li, J. Gong, C. Luo, D. Yao, P. Xu, Structural and functional correlates of motor imagery $\mathrm{BCl}$ performance: insights from the patterns of fronto-parietal attention network, Neurolmage. 134 (2016) 475-485. https://doi.org/10.1016/j.neuroimage.2016.04.030.

[45] J. Decety, F. Michel, Comparative analysis of actual and mental movement times in two graphic tasks, Brain Cogn. 11 (1989) 87-97. https://doi.org/10.1016/0278-2626(89)90007-9.

[46] C. Papaxanthis, T. Pozzo, X. Skoura, M. Schieppati, Does order and timing in performance of imagined and actual movements affect the motor imagery process? The duration of walking and writing task, Behav. Brain Res. 134 (2002) 209-215. https://doi.org/10.1016/s01664328(02)00030-x.

[47] J. Decety, M. Jeannerod, Mentally simulated movements in virtual reality: does Fitts's law hold in motor imagery?, Behav. Brain Res. 72 (1995) 127-134. https://doi.org/10.1016/01664328(96)00141-6.

[48] P. Maruff, P.H. Wilson, J. De Fazio, B. Cerritelli, A. Hedt, J. Currie, Asymmetries between dominant and non-dominant hands in real and imagined motor task performance, Neuropsychologia. 37 (1999) 379-384. https://doi.org/10.1016/s0028-3932(98)00064-5.

[49] P.V. Radulescu, J.J. Adam, M.H. Fischer, J. Pratt, Fitts's Law violation and motor imagery: are imagined movements truthful or lawful?, Exp. Brain Res. 201 (2010) 607-611. https://doi.org/10.1007/s00221-009-2072-2.

[50] J.W. Roberts, T.N. Welsh, C.J. Wakefield, Examining the equivalence between imagery and execution - Do imagined and executed movements code relative environmental features?, Behav. Brain Res. 370 (2019) 111951. https://doi.org/10.1016/j.bbr.2019.111951.

[51] H. Boulton, S. Mitra, Body posture modulates imagined arm movements and responds to them, J. Neurophysiol. 110 (2013) 2617-2626. https://doi.org/10.1152/jn.00488.2013.

[52] R. Gentili, V. Cahouet, Y. Ballay, C. Papaxanthis, Inertial properties of the arm are accurately predicted during motor imagery, Behav. Brain Res. 155 (2004) 231-239.

https://doi.org/10.1016/j.bbr.2004.04.027.

[53] C. Papaxanthis, M. Schieppati, R. Gentili, T. Pozzo, Imagined and actual arm movements have similar durations when performed under different conditions of direction and mass, Exp. Brain Res. 143 (2002) 447-452. https://doi.org/10.1007/s00221-002-1012-1.

[54] M. Baccarini, M. Martel, L. Cardinali, O. Sillan, A. Farnè, A.C. Roy, Tool use imagery triggers tool incorporation in the body schema, Front. Psychol. 5 (2014) 492. https://doi.org/10.3389/fpsyg.2014.00492.

[55] V. Frak, Y. Paulignan, M. Jeannerod, Orientation of the opposition axis in mentally simulated grasping, Exp. Brain Res. 136 (2001) 120-127. https://doi.org/10.1007/s002210000583.

[56] S. Glover, P. Dixon, U. Castiello, M.F.S. Rushworth, Effects of an orientation illusion on motor performance and motor imagery, Exp. Brain Res. 166 (2005) 17-22. https://doi. org/10.1007/s00221-005-2328-4.

[57] C. Calmels, J.F. Fournier, Duration of physical and mental execution of gymnastic routines, Sport Psychol. 15 (2001) 142-150. https://doi.org/10.1123/tsp.15.2.142.

[58] P. Gandrey, C. Paizis, V. Karathanasis, N. Gueugneau, C. Papaxanthis, Dominant vs. nondominant arm advantage in mentally simulated actions in right handers, J. Neurophysiol. 110 (2013) 2887-2894. https://doi.org/10.1152/jn.00123.2013.

[59] M.A. Grealy, G.F. Shearer, Timing processes in motor imagery, Eur. J. Cogn. Psychol. 20 (2008) 867-892. https://doi.org/10.1080/09541440701618782.

[60] K. Hanyu, Y. Itsukushima, Cognitive distance of stairways: a multi-stairway investigation, Scand. J. Psychol. 41 (2000) 63-70. https://doi.org/10.1111/1467-9450.00172. 
[61] B. Cerritelli, P. Maruff, P. Wilson, J. Currie, The effect of an external load on the force and timing components of mentally represented actions, Behav. Brain Res. 108 (2000) 91-96. https://doi.org/10.1016/s0166-4328(99)00138-2.

[62] J. Decety, M. Jeannerod, C. Prablanc, The timing of mentally represented actions, Behav. Brain Res. 34 (1989) 35-42. https://doi.org/10.1016/s0166-4328(89)80088-9.

[63] A.B. Slifkin, High loads induce differences between actual and imagined movement duration, Exp. Brain Res. 185 (2008) 297-307. https://doi.org/10.1007/s00221-007-1154-2.

[64] L. Wong, G.A. Manson, L. Tremblay, T.N. Welsh, On the relationship between the execution, perception, and imagination of action, Behav. Brain Res. 257 (2013) 242-252. https://doi.org/10.1016/j.bbr.2013.09.045.

[65] E. Yoxon, L. Tremblay, T.N. Welsh, Effect of task-specific execution on accuracy of imagined aiming movements, Neurosci. Lett. 585 (2015) 72-76. https://doi.org/10.1016/j.neulet.2014.11.021.

[66] E. Yoxon, S.M. Pacione, J.-H. Song, T.N. Welsh, The action-specific effect of execution on imagination of reciprocal aiming movements, Hum. Mov. Sci. 54 (2017) 51-62. https://doi.org/10.1016/j.humov.2017.03.007.

[67] M.M. Walsh, D.A. Rosenbaum, Deciding how to act is not achieved by watching mental movies., J. Exp. Psychol. Hum. Percept. Perform. 35 (2009) 1481-1489. https://doi.org/10.1037/a0015799.

[68] J. Grèzes, J. Decety, Functional anatomy of execution, mental simulation, observation, and verb generation of actions: a meta-analysis, Hum Brain Mapp. 12 (2001) 1-19. https://doi.org/10.1002/1097-0193(200101)12:1<1::aid-hbm10>3.0.co;2-v.

[69] M. Lotze, U. Halsband, Motor imagery, J. Physiol. Paris. 99 (2006) 386-395. https://doi.org/10.1016/j.jphysparis.2006.03.012.

[70] M. Jeannerod, Motor Cognition: what actions tell the self, Oxford University Press, 2008. https://oxford.universitypressscholarship.com/view/10.1093/acprof:oso/9780198569657.001 .0001/acprof-9780198569657.

[71] K.L. Macuga, S.H. Frey, Neural representations involved in observed, imagined, and imitated actions are dissociable and hierarchically organized, Neuroimage. 59 (2012) 2798-2807. https://doi.org/10.1016/j.neuroimage.2011.09.083.

[72] S. Hétu, M. Grégoire, A. Saimpont, M.-P. Coll, F. Eugène, P.-E. Michon, P.L. Jackson, The neural network of motor imagery: an ALE meta-analysis, Neurosci. Biobehav. Rev. 37 (2013) 930949. https://doi.org/10.1016/j.neubiorev.2013.03.017.

[73] R.M. Hardwick, S. Caspers, S.B. Eickhoff, S.P. Swinnen, Neural correlates of action: comparing meta-analyses of imagery, observation, and execution, Neurosc.i Biobehav. Rev. 94 (2018) 3144. https://doi.org/10.1016/j.neubiorev.2018.08.003.

[74] S. Rossi, M. Hallett, P.M. Rossini, A. Pascual-Leone, Safety of TMS consensus group, Safety, ethical considerations, and application guidelines for the use of transcranial magnetic stimulation in clinical practice and research, Clin. Neurophysiol. 120 (2009) 2008-2039. https://doi.org/10.1016/j.clinph.2009.08.016.

[75] J. Peirce, J.R. Gray, S. Simpson, M. MacAskill, R. Höchenberger, H. Sogo, E. Kastman, J.K. Lindel $\varnothing \mathrm{v}$, PsychoPy2: experiments in behavior made easy, Behav. Res. Methods. 51 (2019) 195-203. https://doi.org/10.3758/s13428-018-01193-y.

[76] D.B. Berch, R. Krikorian, E.M. Huha, The Corsi block-tapping task: methodological and theoretical considerations, Brain Cogn. 38 (1998) 317-338. https://doi.org/10.1006/brcg.1998.1039.

[77] J.R. Wessel, Prepotent motor activity and inhibitory control demands in different variants of the go/no-go paradigm, Psychophysiology. 55 (2018) e12871.

https://doi.org/10.1111/psyp.12871.

[78] S. Monsell, Task switching, Trends Cogn. Sci. 7 (2003) 134-140. https://doi.org/10.1016/S1364-6613(03)00028-7. 
[79] W. Beam, J.J. Borckardt, S.T. Reeves, M.S. George, An efficient and accurate new method for locating the F3 position for prefrontal TMS applications, Brain Stimul. 2 (2009) 50-54. https://doi. org/10.1016/j.brs.2008.09.006.

[80] T.A. Whitten, C.S. Mang, M.S. Cosh, S.H. Scott, S.P. Dukelow, B.W. Benson, Spatial working memory performance following acute sport-related concussion, J. Concussion. 2 (2018). https://doi.org/10.1177/2059700218797818.

[81] S. Glover, P. Dixon, Likelihood ratios: a simple and flexible statistic for empirical psychologists, Psychon. Bull. Rev. 11 (2004) 791-806. https://doi.org/10.3758/bf03196706.

[82] H. Akaike, Information theory and an extension of the maximum likelihood principle, in: B. N. Petrove \& F. Csaki (Eds.), Second International Symposium on Information Theory, Budapest: Academiai Kiado, 1973: pp. 267-281.

[83] J. Cohen, The earth is round ( $p<.05$ ), Am. Psychol. 49 (1994) 997-1003. https://doi.org/10.1037/0003-066X.49.12.997.

[84] G.R. Loftus, Psychology will be a much better science when we change the way we analyse data, Curr. Dir. Psychol. Sci. 5 (1996) 161-171. https://doi.org/10.1111/14678721.ep11512376.

[85] J.P. Simmons, L.D. Nelson, U. Simonsohn, False-positive psychology: undisclosed flexibility in data collection and analysis allows presenting anything as significant, Psychol. Sci. 22 (2011) 1359-1366. https://doi.org/10.1177/0956797611417632.

[86] R.L. Wasserstein, N.A. Lazar, The ASA statement on p-values: context, process, and purpose, Am. Stat. 70 (2016) 129-133. https://doi.org/10.1080/00031305.2016.1154108.

[87] D. Bor, J. Duncan, A.C.H. Lee, A. Parr, A.M. Owen, Frontal lobe involvement in spatial span: converging studies of normal and impaired function, Neuropsychologia. 44 (2006) 229-237. https://doi. org/10.1016/j.neuropsychologia.2005.05.010.

[88] Y.-J. Wu, P. Tseng, C.-F. Chang, M.-C. Pai, K.-S. Hsu, C.-C. Lin, C.-H. Juan, Modulating the interference effect on spatial working memory by applying transcranial direct current stimulation over the right dorsolateral prefrontal cortex, Brain Cogn. 91 (2014) 87-94. https://doi.org/10.1016/j.bandc.2014.09.002.

[89] A.K. Barbey, M. Koenigs, J. Grafman, Dorsolateral prefrontal contributions to human working memory, Cortex. 49 (2013) 1195-1205. https://doi.org/10.1016/j.cortex.2012.05.022.

[90] M. Toepper, H. Gebhardt, T. Beblo, C. Thomas, M. Driessen, M. Bischoff, C.R. Blecker, D. Vaitl, G. Sammer, Functional correlates of distractor suppression during spatial working memory encoding, Neuroscience. 165 (2010) 1244-1253. https://doi.org/10.1016/j.neuroscience.2009.11.019.

[91] W.E. Mackey, O. Devinsky, W.K. Doyle, M.R. Meager, C.E. Curtis, Human dorsolateral prefrontal cortex is not necessary for spatial working memory, J. Neurosci. 36 (2016) 28472856. https://doi.org/10.1523/JNEUROSCI.3618-15.2016.

[92] C.E. Curtis, Prefrontal and parietal contributions to spatial working memory, Neuroscience. 139 (2006) 173-180. https://doi.org/10.1016/j.neuroscience.2005.04.070.

[93] S.R. Paulraj, K. Schendel, B. Curran, N.F. Dronkers, J.V. Baldo, Role of the left hemisphere in visuospatial working memory, J. Neurolinguistics. 48 (2018) 133-141. https://doi.org/10.1016/j.jneuroling.2018.04.006.

[94] H.D. Zimmer, Visual and spatial working memory: from boxes to networks, Neurosci. Biobehav. Rev. 32 (2008) 1373-1395. https://doi.org/10.1016/j.neubiorev.2008.05.016.

[95] M. Rieger, S.F. Dahm, I. Koch, Inhibition in motor imagery: a novel action mode switching paradigm, Psychon. Bull. Rev. 24 (2017) 459-466. https://doi.org/10.3758/s13423-016-10955.

[96] V.K.E. Bart, I. Koch, M. Rieger, Inhibitory mechanisms in motor imagery: disentangling different forms of inhibition using action mode switching, Psychol. Res. 85 (2021) 1418-1438. https://doi.org/10.1007/s00426-020-01327-y.

[97] H. Nakata, K. Sakamoto, A. Ferretti, M. Gianni Perrucci, C. Del Gratta, R. Kakigi, G. Luca Romani, Executive functions with different motor outputs in somatosensory Go/Nogo tasks: 
an event-related functional MRI study, Brain Research Bulletin. 77 (2008) 197-205. https://doi.org/10.1016/j.brainresbull.2008.07.008.

[98] J. Chikazoe, Localizing performance of go/no-go tasks to prefrontal cortical subregions, Curr. Opin. Psychiatry. 23 (2010) 267-272. https://doi.org/10.1097/YCO.0b013e3283387a9f.

[99] U. Mayr, Age differences in the selection of mental sets: The role of inhibition, stimulus ambiguity, and response-set overlap, Psychol. Aging. 16 (2001) 96-109. https://doi.org/10.1037/0882-7974.16.1.96.

[100] I. Koch, M. Gade, S. Schuch, A.M. Philipp, The role of inhibition in task switching: a review, Psychon. Bull. Rev. 17 (2010) 1-14. https://doi.org/10.3758/PBR.17.1.1.

[101] O. Gruber, S. Karch, E.K. Schlueter, P. Falkai, T. Goschke, Neural mechanisms of advance preparation in task switching, Neuroimage. 31 (2006) 887-895. https://doi.org/10.1016/j.neuroimage.2005.12.043.

[102] J.D. Teasdale, B.H. Dritschel, M.J. Taylor, L. Proctor, C.A. Lloyd, I. Nimmo-Smith, A.D. Baddeley, Stimulus-independent thought depends on central executive resources, Mem. Cognit. 23 (1995) 551-559. https://doi.org/10.3758/bf03197257.

[103] S. Funahashi, Prefrontal contribution to decision-making under free-choice conditions, Front. Neurosci. 11 (2017) 431. https://doi.org/10.3389/fnins.2017.00431.

[104] A.D. Baddeley, G. Hitch, Working Memory, in: Psychology of Learning and Motivation, Elsevier, 1974: pp. 47-89. https://doi.org/10.1016/S0079-7421(08)60452-1.

[105] P.S. Holmes, D.J. Collins, The PETTLEP approach to motor imagery: a functional equivalence model for sport psychologists, J. Appl. Sport Psychol. 13 (2001) 60-83. https://doi.org/10.1080/10413200109339004.

[106] C. Wakefield, D. Smith, Perfecting practice: applying the PETTLEP model of motor imagery, J. Sport Psychol. Action. 3 (2012). https://doi.org/10.1080/21520704.2011.639853.

[107] T. Mulder, S. Zijlstra, W. Zijlstra, J. Hochstenbach, The role of motor imagery in learning a totally novel movement, Exp. Brain Res. 154 (2004) 211-217. https://doi.org/10.1007/s00221003-1647-6.

[108] B. Steenbergen, H. Krajenbrink, J.M. Lust, P. Wilson, Motor imagery and action observation for predictive control in developmental coordination disorder, Dev. Med. Child Neurol. (2020). https://doi.org/10.1111/dmcn.14612.

[109] M.W. Scott, G. Wood, P.S. Holmes, J. Williams, B. Marshall, D.J. Wright, Combined action observation and motor imagery: an intervention to combat the neural and behavioural deficits associated with developmental coordination disorder, Neurosci. Biobehav. Rev. 127 (2021) 638-646. https://doi.org/10.1016/j.neubiorev.2021.05.015.

[110] American Psychiatric Association, Diagnostic and statistical manual of mental disorders (5th ed.), Washington, DC (2013).

[111] M. Biotteau, J.-M. Albaret, Y. Chaix, Developmental coordination disorder, Handb. Clin. Neurol. 174 (2020) 3-20. https://doi.org/10.1016/B978-0-444-64148-9.00001-6.

[112] A. Gomez, A. Sirigu, Developmental coordination disorder: core sensori-motor deficits, neurobiology and etiology, Neuropsychologia. 79 (2015) 272-287. https://doi. org/10.1016/j.neuropsychologia.2015.09.032.

[113] S.H. Johnson, Imagining the impossible: intact motor representations in hemiplegics, Neuroreport. 11 (2000) 729-732. https://doi.org/10.1097/00001756-200003200-00015. 